\title{
LE IMMAGINI DI SEbASTIANo VASSALLI DAL PROFONDO DEL TEMPO
}

\author{
Jiří PELÁN \\ Università Carolina di Praga
}

\begin{abstract}
En): In the extensive literary production of Sebastiano Vassalli (1941-2015), we can witness an evolution of his poetics from his initial formation within the avant-garde Gruppo 63 towards a more traditional prose. In this change of perspective, Vassalli's tendency to include in his narratives a documented attention to real historical events, within which his characters move, each following his own original destiny, played a fundamental role. The literary references, in Italy and abroad, from which Vassalli could draw are numerous and well known (Manzoni, Leopardi, Pirandello, Sciascia, Queneau, García Marquez...). On the strength of this methodology, Vassalli has been able to tell stories where the fulcrum of the plots always revolves around his interest in the various human types who have remained unchanged beyond historical periods and who face adverse events in a heroic way.
\end{abstract}

\begin{abstract}
It): Nell'estesa produzione letteraria di Sebastiano Vassalli (1941-2015), si può assistere a un'evoluzione della sua poetica dall'iniziale formazione all'interno dell'avanguardia del Gruppo 63 verso una prosa più tradizionale. In questo mutamento di prospettiva, ha giocato un ruolo fondamentale la tendenza di Vassalli a voler includere nelle sue narrazioni una documentata attenzione verso i reali eventi storici, all'interno dei quali i suoi personaggi si muovono seguendo ognuno il proprio originale destino. I riferimenti letterari, in Italia e all'estero, da cui Vassalli poté attingere sono numerosi e ben noti (Manzoni, Leopardi, Pirandello, Sciascia, Queneau, García Marquez...). Forte di questa metodologia, Vassalli ha saputo raccontare storie dove il fulcro delle trame ruota sempre attorno al suo interesse nei confronti delle diverse tipologie umane rimaste immutate al di là dei periodi storici e che affrontano in modo eroico gli accadimenti avversi.
\end{abstract}

Key-words (En): Sebastiano Vassali; Gruppo 63; postmodern literature; novel; historical events

Parole-chiave (It): Sebastiano Vassalli; Gruppo 63; letteratura postmoderna; romanzo; eventi storici

DOI : $10.32725 /$ eer.2021.015

Uno dei tratti caratteristici della letteratura cosiddetta «postmoderna» è la profonda avversione ad inserire gli avvenimenti narrati all'interno di una cornice storica, tendenza motivata dalla sfiducia proprio nei confronti della storia. La posizione di Sebastiano Vassalli (1941-2015) a questo riguardo non è netta. È vero che condivideva il rifiuto delle «grandi narrazioni», ma il passato in quanto tale lo affascinava nel vero senso del termine. Questa fascinazione ha determinato quindi un'aperta polemica con lo scetticismo postmoderno: la sua vasta opera, i suoi tanti romanzi, sono in linea di principio incentrati sui destini umani nella loro correlazione col movimento della storia, divenendo dunque «storie nella storia».

All'inizio della propria carriera letteraria però Vassalli si era cimentato più volte nella pratica postmoderna di giocare con le parole e coi fatti. Nel 1967 aveva partecipato, a Fano, all'incontro della neoavanguardia raccolta nel Gruppo '63 e per un breve periodo (giusto il tempo che ancora restava all'avanguardia) aveva legato la propria attività creativa al programma del Gruppo. Aveva debuttato nel 1968 con 
la raccolta di testi sperimentali Disfaso (titolo che rimandava alla disfasia, all'incapacità di ordinare in modo appropriato le parole in una frase), a cui erano seguiti in rapida successione tre testi in prosa, che evidenziavano una distanza radicale dalle forme letterarie standardizzate e riducevano l'enunciato a una combinazione verbale sulla scorta della tradizione futuristico-surrealista: Narcisso (1968), Tempo di massacro (1970) e Il millennio che muore (1972) ${ }^{1}$. Solo dopo che l'onda della neoavanguardia aveva cominciato il suo riflusso, Vassalli iniziò a scrivere libri più adeguati alle sue reali disposizioni di scrittore. Il punto di svolta è rappresentato da tre romanzi che tornavano chiaramente ai valori tradizionali della grande prosa, all'intreccio, al disegno psicologico, alla descrizione dell'ambiente: L'arrivo della lozione (1976), Abitare il vento (1980), Mareblù (1982).

Il distacco dalle poetiche della neoavanguardia era già molto chiaro in questi testi, per quanto non ancora esplicito. Degli esperimenti verbali di un tempo Vassalli aveva conservato qui il senso marcato della persiflage linguistica e della parodia, e nelle scelte tematiche risuonava ancora la recente atmosfera intellettuale reideologizzata: il protagonista del primo romanzo era una proiezione del neofascismo, il protagonista del secondo era il ritratto del rivoluzionarismo di sinistra, quello del terzo era la caricatura del comunismo dogmatico di tipo stalinista. Non erano ancora «romanzi storici», certo, ma erano testi comunque molto vicini alla definizione del genere: in tutti e tre i casi il terreno di coltura da cui queste storie si erano sviluppate era costituito dalle recenti peripezie politiche.

Il distacco di Vassalli dalle teorizzazioni e dalla pratica della neoavanguardia nel corso degli anni Settanta arrivò fino al loro rifiuto categorico, espresso poi esplicitamente nel caustico pamphlet Arkadia, pubblicato nel 1983. In questo testo - traboccante di quell'appassionata verve polemica che sarà anche in seguito, con diversa intensità, uno degli ingredienti dei suoi libri - Vassalli definiva gli autori della neoavanguardia, che mettevano in pratica ciecamente le poetiche del gruppo inventate a freddo, eredi degli arcadici del XVIII secolo che si travestivano tutti quanti da pastori per cantare inesistenti Silvie e Fillidi. Riteneva certo lodevoli le «esigenze [...] di aggiornamento culturale dopo la dittatura mussoliniana e dopo gli anni tristi del dopoguerra», ma constatava che «l'avanguardia del Gruppo '63 fu un'avanguardia grigia e impiegatizia, fatta quasi tutta di professori e di funzionari di case editrici.» ${ }^{2}$ Vassalli non rivide mai questa critica sarcastica ${ }^{3}$, e si può essere

\footnotetext{
${ }^{1}$ Per gli esordi di Vassalli tra le fila della neoavanguardia si veda soprattutto Roberto Cicala, «La sperimentazione del giovane Vassalli, (con bibliografia 1965-1984, catalogo delle sue edizioni CDE e Ant. Ed. immagini e testi», in: Roberto Cicala, Inchiostri indelebili. Itinerari di carta fra bibliografie, archivi e editoria, Milano, Educatt 2012, p. 215-248.

${ }^{2}$ Sebastiano Vassalli, Arkadia: carriere, caratteri, confraternite degli impoeti d'Italia, Bergamo, El Bagatt («Pamphlet» n. 1), 1983, p. 12.

${ }^{3} \mathrm{Al}$ contrario, vi è tornato continuamente. Si vedano ad es. le affermazioni contenute nel libro di interviste Giovanni Tesio - Sebasiano Vassalli, Un nulla pieno di storie, Novara, Interlinea 2010, p. 42 : «...era una non-avanguardia, un non-gruppo, un non-tutto-e-il-contrario-di-tutto, tenuto insieme dalle idee confuse ma forti di quegli anni. Era la squadra che si era formata spontaneamente per una partita, che in Italia si gioca dai tempi di Dante Alighieri e dello Stil Novo: la partita dei moderni contro gli antichi'.»Ibidem, p. 44: «la cifra stilistica di Sanguineti, già in quell'epoca, era il cupio dissolvi: la letteratura che si fa in odio alla letteratura. Come il capitalista Giangiacomo Feltrinelli $-30-$
} 
certi che il tono eccitato di questo pamphlet tradiva un risentimento personale, motivato dalla consapevolezza che l'essersi lasciato convincere dalle teorie dell'avanguardia lo aveva sviato dalla sua strada e gli aveva fatto perdere del tempo inutilmente ${ }^{4}$.

Negli anni Ottanta Vassalli iniziò decisamente a volgersi alle concrete «storie umane» che si realizzano in un tempo concreto. Questo interesse è stato senza dubbio il motivo per una serie di pubblicazioni in cui è stata chiaramente data la priorità a una letteratura documentaria, più aderente ai fatti (sia come frutto di studio negli archivi, che di ricognizione «sul terreno») rispetto alla libera fabulazione. Per prima cosa pubblicò La notte della cometa (1984), una biografia del poeta Dino Campana splendidamente documentata. Su richiesta di Giulio Bollati, si recò poi in Alto Adige per predisporre per la rivista «Panorama» una mappa della situazione di un territorio diviso per etnie e amministrato (col silenzioso assenso di Roma) dalla maggioranza tedesca locale; il risultato fu il provocatorio documento Sangue e suolo. Viaggio tra gli italiani trasparenti $(1985)^{5}$. Una ricerca documentaria era alla base anche del saggio L'Alcova elettrica (1986), nel quale ha trattato la storia del processo del 1913 contro la rivista futurista «Lacerba», provocato dall'articolo di Tavolato «Elogio della prostituzione».

Il romanzo $L$ 'oro del mondo (1987) è stato in modo notevole la risultante di una ricerca ventennale. Vassalli vi mise in pratica per la prima volta la poetica narrativa nella quale si era definitivamente ritrovato e che doveva diventare in gran parte il paradigma formale dei testi che si sarebbero riallacciati immediatamente a questo romanzo: una poetica che con evidente leggerezza combina la vicenda narrata con una minuziosa documentazione dei fatti. L'oro del mondo narra innanzitutto il destino di un uomo - la storia della vita di un ragazzo che cresce nel periodo della transizione storica che va dalla caduta del fascismo ai primi anni della ripresa postbellica. Al filo di questo destino (su un evidente sfondo autobiografico) sono poi legate altre storie - del padre del ragazzo, di sua madre, di suo zio e di molte altre comparse - presentate in genere con un breve aneddoto, ma comunque in maniera abbastanza plastica, così che sia evidente che anche queste storie meritano

faceva la rivoluzione proletaria contro sé stesso, anche lo scrittore, secondo Sanguineti, doveva dimostrare con le sue opere che la letteratura è morta, e che fa schifo.»

${ }^{4}$ Lo ha sostenuto lo stesso Vassalli nell'intervista con Maurizio Zuccari: «...io sono diventato scrittore a quarant'anni, perché prima c'erano questi sperimentalismi. Ecco, prima ho detto di non credere ai geni, se lo fossi stato a vent'anni avrei detto: no all'avanguardia, agli sperimentalismi. Il mio mestiere è raccontare storie e avrei cominciato da allora, invece ho seguito un percorso più tortuoso. Insomma, è una questione generazionale, comunque io sono diventato scrittore dopo i quarant'anni. Non rifarei nulla di quello che ho fatto, sono due autori completamente diversi. D'altra parte si è figli della propria epoca, si appartiene ad una generazione. Se io non avessi fatto determinate esperienze probabilmente non sarei diventato neppure scrittore e non avrei cominciato a pubblicare. Chi ha la mia età doveva fare quel percorso lì. Se lei guarda, oggi di scrittori della mia età non ce n'è quasi, sono quasi tutti della generazione prima o dopo, la mia età è stata bruciata proprio perché chi ha cominciato come me con queste storie, il Gruppo '63, gli esperimenti formali, poi ha continuato lì ed è miseramente finito. Evidentemente io avevo qualche ragione diversa e maggiore che mi ha permesso di riciclarmi, cosa che non ha fatto quasi nessuno.» «Intervista di Maurizio Zuccari con Sebastiano Vassalli: Lo scrittore e la storia», dicembre 2003, tratta dal sito http://www.ragionamentidistoria.it

${ }^{5}$ Dopo due decenni il tema è stato ripreso nel saggio storico Il confine, Milano, Rizzoli, 2015.

$$
-31 \text { - }
$$


di essere narrate. Ognuna di esse, cioè, rappresenta il tassello di quell'enorme e immenso mosaico che viene appunto chiamato «storia» (nel romanzo di Vassalli queste immagini frammentarie illustrano in concreto il periodo della dittatura fascista, quello del passaggio alla democrazia e dell'impetuoso inizio degli istinti consumistici nel corso degli anni Cinquanta).

Già in questo testo sono presenti alcune premesse che diverranno in seguito caratteristiche della sua opera. Vassalli non spaccia la lanterna magica dei suoi romanzi per la «verità» sulla storia e non si atteggia a moralista che apporta il suo insegnamento per il futuro. Già qui la sua visione della storia ha un carattere per nulla patetico. La storia per lui non è una cosa che si possa racchiudere all'interno di schemi sensati, e soprattutto non è affatto l'illustrazione di un «progresso» lineare, prestabilito. La storia - fa intendere Vassalli - in realtà non si può neppure raccontare; si possono raccontare solo le singole vicende umane che si confrontano con l'irrazionalità della storia: si può solo chiedere come si comportano gli uomini sulle quinte della storia. Ed è certo che esistono delle storie che sono più interessanti di altre, e questo viene fuori maggiormente quando si parla dei sogni che gli uomini hanno in determinati momenti storici, sia che vi aderiscano, sia che vi entrino in contrasto.

Già dal suo L'oro del mondo risuona la convinzione che finché accumuleremo dentro la cornice di un'epoca storica sufficienti vicende come questa, potremo arrivare anche a qualcosa di più generale, a qualcosa che parli della mentalità collettiva di una comunità, di una entità nazionale. Ciò che interessa maggiormente Vassalli è quel fenomeno unico che si chiama «carattere nazionale» ${ }^{6}$. Le vicende che narra ne L'oro del mondo, tutte inerenti alla conversione in massa degli italiani dal fascismo alla democrazia, testimoniano soprattutto l'enorme capacità di dimenticare da parte di questo concreto collettivo.

Il tratto formale che caratterizza il romanzo $L$ 'oro del mondo è dato dal fatto che Vassalli insieme all'avanguardistico «narrare sul nulla» rifiutò con veemenza di creare l'illusione che l'autore non partecipi a quanto narrato, come la praticava ad esempio il nouveau roman francese. Sebbene stavolta nella sostanza avesse scritto già un romanzo «storico» - anche se si tratta di storia ancora non troppo remota -, non esitò a inserire nel suo scenario anche il proprio personale hic et nunc, entrandovi come il narratore della storia, un intellettuale degli anni Ottanta, una forza salariata delle case editrici. Ha quindi fatto vedere che quel che leggiamo sono immagini esclusivamente sue, che non possono essere scambiate per impersonali fotografie storiche, e che queste vicende - in quanto parte del collettivo descritto lo riguardano direttamente. Questo procedimento sarà mantenuto anche per le narrazioni che seguono immediatamente e che sono datate in un passato molto più remoto.

Nel romanzo La chimera (1990), che racconta la storia di una giovanissima strega bruciata sul rogo nel 1610, Vassali si è pronunciato con ancora maggiore

\footnotetext{
${ }^{6}$ È uno dei temi centrali di Vassalli: vi è tornato esplicitamente venti anni dopo nella raccolta di commenti giornalistici Gli italiani sono gli altri, Torino, Baldini \& Castoldi, 1998, e nella raccolta di saggi scritti sulla scia dei «caratteri» di La Bruyère“ L'Italiano, Torino, Einaudi 2007.
} 
nettezza a proposito del suo interesse per la storia ${ }^{7}$. Nelle pagine introduttive della narrazione ha espresso il suo dubbio sulla reale possibilità di fornire un'immagine del tempo presente fin tanto che non ci poniamo la domanda sulla natura del suo substrato:

...il presente è più che mai rumore, non contiene niente che meriti di essere raccontato: milioni, miliardi di voci che gridano, tutte insieme, in tutte le lingue e cercando di sopraffarsi l'una con l'altra, la parola 'io'... Per cercare le chiavi del presente, e per capirlo, bisogna uscire dal rumore ${ }^{8}$.

L'ispirazione originaria di questo giudizio è forse pirandelliana (Pirandello per Vassalli era senz'altro uno dei riferimenti fondamentali): vi risuona la fascinazione del passato silenzioso, archiviato, nel quale si immergeva l'Enrico IV di Pirandello. Le parole sul passato in quanto «chiavi del presente» ricordano anche Leonardo Sciascia (un autore che per Vassalli aveva un altrettanto peso specifico) ${ }^{9}$ : proprio Sciascia - soprattutto nei suoi saggi - si volgeva al passato quasi con solennità, per cercare nelle sconfitte passate i motivi della sconfitta di oggi. A livello più profondo, però, il volgersi di Vassalli al passato ha senza dubbio un motivo sostanzialmente più elementare, un motivo che nel modo più straziante aveva già espresso Leopardi (con la cui visione pessimistica della sorte umana Vassalli si identifica a più riprese): la coscienza tragica della distruttività del tempo, che liquida incessantemente le vite individuali con il loro modesto corredo di sentimenti, di gioie e di dolori, nell'interesse del movimento continuo della macchina mondiale. L'attività dello scrittore in questa prospettiva si rivela l'unico modo per porre un argine a questa forza distruttiva. Del resto Vassalli aveva già ricordato questa motivazione del proprio scrivere ne $L$ 'oro del mondo:

Con quel tanto di lucidità che ci rimane guardiamo il tempo che ci strangola, avvolgendoci tra le sue spire, che lentamente ci inghiotte: adesso ancora ci siamo e poi, quando non ci saremo più, nessuno al mondo se ne rammaricherà, non resteranno spazi vuoti a segnalare un'assenza, la nostra assenza. Soltanto, un'altra mela marcita si sarà staccata dall'albero della vita e sarà andata a far strame perché l'albero possa rinnovarsi, continuamente, nel tempo ${ }^{10}$.

Neppure il teatro del romanzo La Chimera (1990), storia della strega Antonia Spagnoletti, il paesino di Zardino nella Pianura padana, esiste più, visto che è stato portato via secoli fa dalle acque di una grande alluvione (quel che oggi al suo posto si può vedere dalla propria automobile viene spiegato nel settimo capitolo). E

\footnotetext{
${ }^{7}$ Sul romanzo si veda Roberto Cicala e Giovanni Tesio (a cura di), La Chimera. Storia e fortuna del romanzo di Sebastiano Vassalli, con testi di Gian Luigi Beccaria, Carlo Bo, Maria Corti, un inedito di Umberto Bellintani e appendice di Sebastiano Vassalli, Novara, Centro Novarese di Studi Letterari Interlinea, 2003.

${ }^{8}$ Sebastiano Vassalli, La Chimera, Torino, Einaudi 1990, p. 11.

${ }^{9}$ Vassalli curò le note per i testi di Sciascia Il giorno della civetta e La scomparsa di Majorana per la collana einaudiana «Letture per la scuola media» (1972, 1981). Cfr. Velania La Mendola, "L'onorevole» e «il giullare di provincia». Incontro e scontro letterario tra Sciascia e Vassalli, Microprovincia 49, gennaio-dicembre 2011, (La parola e le storie di Sebastiano Vassalli. Omaggio per $i$ settant'anni dello scrittore), p. 191-205.

${ }^{10}$ Sebastiano Vassalli, L'oro del mondo, Torino, Einaudi 1987, p. 57.
} 
proprio dal nulla che è rimasto Vassalli ha fatto riemergere nel suo romanzo la storia di una ragazza, una trovatella, che aveva vissuto anni relativamente felici nella famiglia dei genitori adottivi, si era innamorata di un giovinetto che, come decine di altri, viveva una vita raminga (era uno dei cosiddetti camminanti che si rifiutavano di fare vita sociale), e venne infine accusata di aver avuto rapporti intimi col diavolo. Sullo sfondo di questa storia Vassalli ha ricostruito minuziosamente la vita di questo piccolo lembo di mondo a cavallo tra XVI e XVII secolo, di una zona marginale del Milanese allora spagnolo, con la sua monotona quotidianità (descritta dettagliatamente grazie all'utilizzo di decine di espressioni dialettali che non hanno equivalenti nell'italiano letterario). Lo ha popolato con numerose figure le cui vicende personali si toccano, si intrecciano e riscompaiono nell'oblio: accanto al falso prete (in realtà un semplice ostiario) che alleva bachi da seta dentro la chiesa, e il canonico Cavagna, cercatore di reliquie truffato, sulla scena compaiono i portatori delle rigide istanze morali dell'era post tridentina: don Teresio e il vescovo di Novara Carlo Bascapè, allievo di Carlo Borromeo, e assieme a loro una serie di altre figure tipiche dell'epoca (il cavaliere predatore, il boia, etc.). Il mosaico che Vassalli compone è quindi un quadro molto suggestivo di un'epoca che nella memoria storica è molto sfumata, un'epoca piena di crudeltà istintiva e anche ambita: nel secondo caso della crudeltà che portava con sé il sogno di realizzare l'ideale ascetico cristiano, il sogno della cui incarnazione doveva occuparsi l'Inquisizione.

La precisione dei dettagli in molti passaggi pone questo romanzo al confine col saggio storico. Vassalli stesso ha però riconosciuto che il nucleo epico della narrazione - la storia di Antonia Spagnoletti - era del tutto inventato. Nello stesso tempo, però, è del tutto reale: è una storia modello nella quale si sono sommate decine di fatti analoghi storicamente documentabili. Se Vassalli l'ha riempita di personaggi e di una descrizione dei fatti tratta da memorie storiche e da documenti d'archivio $^{11}$, non lo ha fatto per il piacere della mistificazione, ma per una inclinazione consapevole verso un altro dei modelli classici della prosa italiana: quel modello di romanzo storico che sono i Promessi sposi di Manzoni. Proprio Manzoni - sulle orme di Walter Scott - all'inizio del XIX secolo aveva proposto una modifica del romanzo storico, genere di straordinario successo, che evoca con plasticità un determinato momento del passato attraverso la combinazione della vicenda modello di due personaggi immaginari (rappresentanti di una folla di anonimi) con il disegno al microscopio dello sfondo, ancorato a materiale documentario verificato (provato da relazioni storiche). Per quanto nel contesto postmoderno potesse avere un effetto quasi provocatorio, Vassalli non ha minimamente nascosto di aver fatto propria questa eredità classica: riconosceva apertamente che il suo dialogo col passato aveva anche questa - letteraria - dimensione. Da essa, del resto, non ha ripreso soltanto la costruzione narrativa, ma anche l'ethos proprio delle vicende tramandate: anche le

\footnotetext{
${ }^{11}$ Vassalli intervistato da Nicola Fano, L'Unità, 25. 1. 1990: «Per La Chimera ho lavorato quattro anni, cercando documenti un po' dovunque. Così pure per L'oro del mondo, per La notte della cometa. E così farò anche per il prossimo romanzo.»
} 
sue storie erano soprattutto un apologia, non cattolica stavolta - a differenza di Manzoni - ma laica, degli «umiliati e offesi».

Non meno marcata è la presenza di questo grande modello anche nel successivo romanzo Marco e Mattio (1992). Anche le vicende di questo romanzo - una delle vette dell'opera di Vassalli - sono inserite con cura all'interno di concrete coordinate geografiche e storiche. Il luogo dell'azione è la regione montuosa tra Venezia e Belluno, il tempo è il periodo a cavallo tra XVIII e XIX secolo. La ratio illuministica non ha accesso in questa contrada dimenticata da Dio (e se ci è arrivata per sbaglio, manifesta il suo volto nascosto: suo portatore è un diabolico avventuriero, don Marco, truffatore e assassino). Il sopruso e la violenza, prodotto tipico dell'agire umano presente in tutte le epoche, anche qui trova senza difficoltà una strada, a cominciare dagli amministratori veneziani e dai soldati napoleonici, fino ai temporanei occupanti austriaci. Vassalli ridà vita a questo mondo scomparso con insaziabilità barocca attraverso decine di comparse di notevole interesse: accanto al prete avaro incontriamo la santa locale, vittima dell'anoressia, falsari, briganti, operai inselvatichiti di fucine in rovina, minatori e carbonai. Il cromatismo di questo brutale microcosmo è infinito ed è anch'esso descritto con precisione, col suo linguaggio peculiare. In questa cornice quindi Vassalli ha inserito la storia di Mattio, un povero sempliciotto, un calzolaio di campagna perseguitato senza tregua dal pensiero importuno della propria purificazione e della salvezza del mondo. Mattio, nel quale il prete del luogo, un ex castrato dalla voce stupenda, nella sua mezza follia aveva riconosciuto il nuovo Cristo, si castra anche lui di propria volontà e alla fine, con inventiva sovrumana, si crocefigge da sé. Finisce sull'isola di San Servolo, in uno dei primi manicomi d'Europa, per incontrarsi con la figura contrappuntistica di don Marco, mefistofelico Ahasver, che incarna il rovescio di tutti gli umani sogni sulla purezza angelica capace di riscattare il mondo.

Le simpatie di Vassalli in questa storia - come già nella Notte della cometa sono rivolte tutte ai «pazzi», ai quali il romanzo è pure dedicato. Nell'ironica conclusione si constata che l'energia dissipata dai folli redentori del mondo non si perde e sebbene non sia in grado di raggiungere il proprio scopo, agisce attraverso vie inesplorate per un relativo miglioramento delle sorti umane:

Insomma e per farla breve, da quel lontano giorno d'aprile del 1806 tutto nel mondo incominciò a volgere al meglio, cioè al presente: a questo nostro presente pieno di cibo, di soldi, di automobili e d'ogni altro genere d'abbondanze, che non sarebbe com'è, o, forse, non esisterebbe nemmeno, se Mattio Lovat non avesse patito, e non fosse morto, per liberarci dal passato $^{12}$.

Nei suoi romanzi Vassalli esprime ripetutamente questa fiducia nelle piccole e imponderabili azioni di sognatori solitari. E questo malgrado il fatto che la sua visione della storia sia per principio ispirata alla disillusione e che registri nel movimento storico - così come la protagonista de La Chimera - solo la manifestazione di «un'energia insensata, una mostruosa malattia che scuote il

${ }^{12}$ Sebastiano Vassalli, Marco e Mattio, Torino, Einaudi 1992, p. 313-314. $-35-$ 
mondo e la sostanza stessa di cui sono fatte le cose», mentre tutti gli ideali, «la giustizia, la legge, Dio, l'Inferno - sono storie più fragili d'un sogno» ${ }^{13}$. Il messaggio che fornisce la letteratura, del resto, per Vassalli è profondamente determinato proprio da questo fatto: se nella storia umana troviamo soprattutto orrore e sofferenza, allora anche alla letteratura è prescritto fin dai tempi di Omero di orientarsi alla loro riproduzione. Così ne parlò Vassalli in una lettera ad Attilio Lolli:

La letteratura si nutre di cose orrende: le persecuzioni razziali, i campi di sterminio, lo sfruttamento dell'uomo sull'uomo, i massacri delle guerre e le minute prevaricazioni della vita quotidiana sono i suoi bignè, il suo terreno ideale di coltura, il suo brodo primordiale ${ }^{14}$.

Malgrado ciò, è strano quanto spesso i protagonisti delle prose di Vassalli siano proprio coloro che si sono ribellati al cieco corso della «storia»: individui ingenui nei quali è come se si replicasse in continuazione la figura del poeta a cui Vassalli ha dedicato tante attenzioni e tanto affetto - la figura di Dino Campana, il «babbo matto»-, individui che non ce la fanno a non inseguire il sogno, l'anelito, l'idea di un modo alternativo di essere. Il loro sogno in verità si scontra, inarrestabile, con la realtà e sono quindi regolarmente predestinati alla sconfitta: proprio questi «vinti» però agli occhi di Vassalli sono i veri eroi, di cui vale la pena narrare le storie. Così è ad esempio nel romanzo Stella avvelenata (2003), anch'esso rivolto a un passato abbastanza remoto e che narra la storia del giovane chierico Leonardo Scacco, il quale nel 1441 parte per andare a studiare a Parigi, strada facendo però si imbatte in una setta di eretici in cerca del mondo perfetto, della leggendaria Atlantide. Scacco si unisce alla loro comitiva e giunge peregrinando sulle coste dell'America; anche lui però alla fine scopre che i «protouomini» che qui incontra sono predisposti alla menzogna e alla violenza quanto quelli che aveva conosciuto in patria, e che ogni stella dell'utopia è avvelenata. Nel suo ultimo romanzo, Io Partenope (2015), che evoca di nuovo il mondo del barocco europeo, è interpretata la storia di Giulia di Marco, una donna che mostrava al prossimo come parlare con Dio attraverso l'anima e il corpo, che però il tribunale dell'Inquisizione scoprì essere un'eretica che con le sue eresie aveva contagiato tutta Napoli.

L'interesse di Vassalli per epoche più remote (XV, XVI e XVII secolo) è molto vasto, però una serie di suoi romanzi è dedicata anche alla storia più recente, ai secoli XIX e XX. E proprio con questi romanzi che Vassalli vuole parlare ai suoi coetanei nel modo più diretto. Se tralasciamo L'oro del mondo, non del tutto «storico», il primo di questa serie è stato Il Cigno (1993). La geografia di questo

\footnotetext{
${ }^{13}$ La Chimera, op. cit., p. 292.

${ }^{14}$ Sebastiano Vassalli - Attilio Lollini, Belle lettere, Torino, Einaudi 1991, p. 51. Vassalli si ritrova qui su una posizione che prima di lui aveva già sostenuto Raymond Queneau nel suo saggio pessimista L'histoire modèle (scritto nel 1942, pubblicato a Parigi, Gallimard 1966), quando dichiarò che se non ci fosse l'infelicità, non ci sarebbe di che raccontare, poiché «le bonheur est homogène, le malheur est divers» (p. 15). Anche la visione della storia di Queneau è molto prossima a quella di Vassalli: «L'histoire ne permet pas de prévoir, d'agir, de modifier les événements. Elle n'est pas une science. Elle demeure au stade qualificatif, alchimique, astrologique. Elle est un simple récit...» (ibidem, p. 10).
} 
romanzo è spostata nel sud d'Italia, e già questo provoca una certa sorpresa (uno scrittore del Nord che scrive del Sud in Italia viene sospettato a priori di incompetenza). Vassalli in questo caso seguì apertamente le tracce del Pirandello da lui più ammirato (in concreto, del Pirandello autore de I vecchi e i giovani, quadro panoramico dell'unificazione d'Italia vista con gli occhi del Sud) e anche quelle di Leonardo Sciascia (autore dei romanzi Il giorno della civetta e A ciascuno il suo), per rievocare una storia semidimenticata della cronaca politica italiana: l'assassinio del marchese Emanuele Notarbartolo - sindaco di Palermo e direttore generale del Banco di Sicilia -, commissionato dal deputato Raffaele Palizzolo, uno dei capi della nascente mafia siciliana.

La scelta del tema anche in questo caso non era stata congiunturale: appassionato alla questione della misura in cui le luci e le ombre del passato condizionano il nostro presente, Vassalli doveva quasi necessariamente affrontare il tema della mafia. Il dibattito provocato dal romanzo costrinse Vassalli a pronunciarsi esplicitamente sul tema in una serie di articoli sui giornali che alla fine raccolse in due sezioni dell'interessante selezione dei suoi interventi polemici dal titolo Gli italiani sono gli altri, accompagnato dall'eloquente sottotitolo Viaggio [in undici tappe] all 'interno del carattere nazionale italiano (1998). Nei suoi commenti in margine al libro, Vassalli ha indicato la vicinanza di questo testo al precedente L'oro del mondo: anche la storia della mafia per certi aspetti è una prova di come l'indole nazionale degli italiani dimentica pericolosamente ciò che non vuole ricordare. La vecchia storia rievocata nel romanzo già a suo tempo aveva dimostrato quasi interamente le pratiche della mafia e il suo collegamento con la sfera politicoeconomica; una precisa analisi della mentalità mafiosa si era potuta poi già leggere nel libro del deputato repubblicano Napoleone Colajanni Nel Regno della mafia del 1900 o nelle memorie di Leopoldo Notarbartolo, il figlio dell'ucciso, del 1911 (quindi in libri da cui Vassalli attinse per la sua ricostruzione romanzata). Già in questi testi vecchi ormai quasi un secolo, Vassalli aveva ritrovato le caratteristiche precise del Sud dell'Italia (che per la corta memoria nazionale è stato necessario riscoprire ogni volta, e spesso a costo di vite umane): la terra - come aveva scritto Leopoldo Notarbartolo - «in cui il pubblico utile non è mai concepito come base dell'utile privato, ma in antagonismo a questo» ${ }^{15}$ e dove - come aggiunge Vassalli - il provinciale «spirito di clan», l'amore dei Siciliani per i Siciliani, spacciato per patriottismo, è garanzia di intoccabilità anche per coloro che l'opinione pubblica altrimenti considera generalmente colpevoli. Ai suoi occhi la storia che ha narrato nel romanzo acquistava una valenza particolarmente memorabile perché per la prima volta nella storia della Sicilia tutta l'isola (ma anche della sezione siculoamericana) si unì nell' organizzazione «Pro Sicilia» per ottenere dalla giustizia dello Stato la liberazione del proprio eroe, il deputato Palizzolo, condannato a undici anni di reclusione, lo portato in trionfo per le strade di Palermo e subito dopo completamente dimenticato - troppo compromesso e ormai impotente. Vassalli scrisse questo romanzo come forma di protesta contro la «cultura» mafiosa, secondo lui glorificata involontariamente anche da coloro i quali ne denunciavano gli effetti

${ }^{15}$ Citato da Sebastiano Vassalli, Gli italiani sono gli altri, Milano, Baldini \& Castoldi 1998, p. 37.

$$
-37-
$$


nefasti (e Vassalli lo rimproverava anche a Sciascia), e con la quale lo Stato italiano (da Francesco Crispi, uno dei protagonisti del romanzo, fino a Giulio Andreotti) ha sempre cercato una qualche forma di accordo, senza mai aver dimostrato di opporre a questa «cultura» un'alternativa positiva che fosse attraente per tutta la comunità nazionale.

Sebbene per la dettagliata aderenza a vicende documentate Il Cigno (il titolo è il nomignolo di don Palizzolo) sia vicino alla letteratura documentaria, il suo statuto di romanzo resta al di fuori di ogni dubbio: Vassalli ha creato anche qui con invidiabile leggerezza un universo immaginario riccamente popolato che è un theatrum mundi del tutto convincente. Egli chiedeva a buon diritto che venisse riconosciuta la «natura romanzesca» di quest'opera, e ricordava di essere stato il primo ad avere abbandonato lo schema del romanzo (pseudo)poliziesco che fino ad allora era stato uno schema obbligato per la tematica mafiosa.

Forse per dimostrare che la sua capacità fabulatoria non era diminuita, subito dopo scrisse un romanzo in cui diede libero corso alla sua fantasia: il provocatorio apocrifo satirico-utopistico 3012, col sottotitolo L'anno del profeta (1995), ${ }^{16}$ nel quale ha tratteggiato una possibile variante del futuro del genere umano (anche qui si impone come possibile fonte di ispirazione il nome di Leopardi, l'autore delle allegoriche Operette morali). Da una distanza di due millenni si commenta qui il libro sacro che aveva registrato le parole e gli atti di Antal, un profeta assassinato nel 3012. Considerato che il profeta aveva trascorso la sua giovinezza in Italia (in quel megavillaggio turistico felliniano che copre il territorio che un tempo andava da Rimini a Cattolica), è comunque necessario decifrare anche alcuni elementi e oggetti di una civiltà risalente ai tempi bui del XXsecolo (le bizzarre elezioni politiche di questa nazione di cuochi e cantanti magnifici che non era capace di governarsi, gli elementi preferiti del suo folklore, come ad esempio i cosiddetti «scioperi» e così via). Attraverso il prisma di questi frammentari ritorni in senso contrario al corso del tempo, emerge anche qui una prospettiva storicizzante.

In seguito comunque Vassalli torna a un modello di romanzo che aveva già sperimentato, sottoponendolo a un'ingegnosa modifica. Il ruolo di protagonista del romanzo Cuore di pietra (1996) non lo fa interpretare da un personaggio umano, ma da una casa borghese in una città della provincia piemontese ai piedi del Monte Rosa. Lo spazio narrativo viene poi riempito - a mo' di un album di famiglia - con le decine di storie degli inquilini che si sono succeduti. Questa idea gli ha permesso di realizzare in modo straordinariamente efficace ciò a cui tendeva già con L'oro del mondo: con la passione di un entomologo ha fissato sulla carta il comportamento dei più svariati tipi umani su uno sfondo storico in movimento, che va dall'Unità d'Italia fino agli anni Settanta del XX secolo. Gli echi critici a questo romanzo dedicato «ai miei lettori, inquilini consapevoli della casa del tempo» ${ }^{17}$ - sono stati molto positivi: apprezzavano soprattutto come Vassalli era riuscito a trasformare una lunga serie di miniritratti individuali in uno schermo panoramico, nel quale si

\footnotetext{
${ }^{16}$ Cfr. Cristina Nesi, Sebastiano Vassalli, Fiesole, Cadmo 2005, p. 90: «Non possiamo escludere che, a determinarne l'esigenza [di scrivere sul futuro], sia stato proprio un irriverente bisogno di sbeffeggiare chi gli aveva 'contristato l'anima per anni con l'etichetta di romanziere storico'.»

${ }^{17}$ Sebastiano Vassalli, Cuore di pietra, Torino, Einaudi, 1996, p. 1. 
integravano «il reale e l'immaginario» (Alfredo Giuliani) e in cui le minuscole tessere del mosaico alla fine si componevano in un «corso o controcorso di storia contemporanea» (Lorenzo Mondo) ${ }^{18}$.

Il romanzo inizia con la nascita di una grande casa costruita per don Basilio Pignatelli da un architetto megalomane. Sulle vicende di don Basilio e dei suoi figli viene poi dimostrata la democratizzazione della società nell'era positivistica, la conseguente crescita della ricchezza e il nuovo attacco dell'irrazionalità (tutti questi sintomi si registrano anche nella topografia della città con la nascita di nuovi edifici: i bagni pubblici, la casa municipale, la banca e il manicomio). I figli del portiere, ex garibaldino, diventano socialisti: uno di loro muore durante il massacro di Milano, un altro parte per l'America, terra atipica, dove «i poveri non si portavano dietro quella maledizione di dover cambiare il mondo»" 19 . Uno dei nipoti di don Basilio, sull'onda del sogno coloniale italiano, parte per l'Africa. Le altre vicende che da qui si ramificano senza sosta accompagnano il lettore attraverso la Prima Guerra mondiale, l'era del fascismo e della Seconda Guerra mondiale (con l'appendice della Repubblica sociale di Mussolini). Dopo la morte dell'ultima erede della famiglia Pignatelli, la casa passa in mano al comune: nelle sue singole stanze ci sono la sede di un'organizzazione politica, di una scuola di ballo, di portatori di handicap, ci si tengono le prove di un gruppo rock; una stanza viene affittata da una appartenente alle «Brigate Rosse», ultima discendente dei «rivoluzionari scientifici» che erano impazziti perché nessuno voleva condividere i loro sogni; alla fine la casa diventa un rifugio di immigrati clandestini provenienti da ogni angolo del mondo.

Con Cuore di pietra Vassalli ha scritto un romanzo che per diversi aspetti - e non solo per l'ampiezza temporale - ricorda Cent'anni di solitudine, la saga latinoamericana di Gabriel García Márquez. Anche qui un determinato collettivo è messo a confronto con il movimento della storia, anche qui diverse eredità si iscrivono senza sosta nei volti e nei destini delle generazioni che si susseguono. A differenza del mondo di Marquez però qui non c'è niente di magico; anche i motivi più bizzarri (il cadavere nel sotterraneo, la collezione di animali esotici che inonda la casa dopo la morte del viaggiatore africano, i furori erotici della parente siciliana, l'assassinio del neonato) si confondono facilmente con la prosaicità e la banalità. Il romanzo di Vassalli è in realtà ancora una volta vicino ai grandi affreschi nazionali, come il già citato I vecchi e i giovani di Pirandello oppure Il gattopardo di Tommasi di Lampedusa. Lo è non solo per la valutazione scetticamente sobria della realtà postunitaria, ma anche per lo sguardo disilluso sui comportamenti umani presenti sul palcoscenico della vita. Uno dei figli di don Basilio nel romanzo dice:

Le generazioni si incalzano nel mondo come si incalzano i piedi dei ballerini seguendo le note del valzer; e ogni generazione riempie il suo presente con quei sogni, che le appartengono di diritto e che lei sola è destinata a sognare ${ }^{20}$.

${ }^{18}$ Formulazioni ricordate nel risvolto della quarta pagina di copertina. Per le recensioni di Lorenzo Mondo ai romanzi di Vassalli, si veda ora: Lorenzo Mondo, Questi piemontesi. Profili di scrittori italiani tra Otto e Novecento, Firenze, Leo S. Olschki 2015.

${ }^{19}$ Sebastiano Vassalli, Cuore di pietra, op. cit., p. 94-95.

${ }^{20}$ Sebastiano Vassalli, Cuore di pietra, Torino, Einaudi 1996, p. 102-103. - 39 - 
Vassalli ammette nello stesso tempo la sua nostalgia per la grande epica, che fino ad allora aveva potuto fondarsi su una visione mitica del mondo: gli antichi dei si chinano sopra un paesetto di provincia e prorompono a ogni istante in una risata omerica, ma ormai da tempo non si immischiano più nella commedia umana ${ }^{21}$. Non si può comunque negare una certa arcaica dimensione epica alla descrizione di Vassalli: essa nasce dal fatto che il tema principale di questo romanzo - come pure dei precedenti, come abbiamo già potuto notare - in realtà, e ancora una volta, non è neppure tanto la storia, quanto piuttosto un tempo percepito - leopardianamente in maniera non antropocentrica:

È lui che ci parla con la nostra voce, che ci guida, che manipola i nostri desideri e i nostri sogni e alla fine cancella le nostre vite per sostituirle con altre vite, di altri uomini che noi non conosceremo mai. ${ }^{22}$

E di nuovo: solo la letteratura è il luogo privilegiato in cui le nostre vite possono lasciare una traccia.

Sette giorni di febbre e due giorni di agonia, e la signorina Orsola se ne andò da questa affollata anticamera che è il mondo dei vivi, per trasferirsi nell'unico mondo durevole e reale: quello dei personaggi di romanzo ${ }^{23}$.

Per 1'ultima volta Vassalli ha ripreso una saga corale alla Márquez nel suo romanzo migliore: Le due chiese, nel quale è descritta la vita di una comunità contadina nei contrafforti piemontesi delle Alpi per un lungo arco di tempo che va dagli anni precedenti la Prima Guerra mondiale fino ai giorni nostri. Nel corso di questo periodo anche in questo libro si succedono alcune generazioni e i bizzarri nomignoli dialettali dei singoli personaggi aprono degli spiragli sul passato profondo. Il tempo dell' isolata comunità pedemontana, regolato dagli atavismi, dai pregiudizi, dalle forme abitudinarie - simili a quelli che Vassalli aveva già descritto nei romanzi Marco e Mattio e La Chimera -, è all'apparenza immobile e si manifesta come semplice canovaccio di vite che hanno il destino già segnato una

\footnotetext{
${ }^{21}$ Nelle interviste Vassalli ha ripetutamente manifestato questa nostalgia per la «grande epica»: «prima di Ulisse c'erano la morte, la vita, la violenza cieca, la lussuria, l'incesto: un mondo in cui trionfavano gli istinti animali, l'uomo era a metà strada del suo percorso tra l'essere animale e l'essere sociale e civile che sta ancora cercando di diventare, però non poteva avere delle storie perché tutto si risolveva in questi episodi: uno incontrava un altro e lo accoppava, incontrava una donna e se la ingroppava. Nasceva, moriva, si ubriacava, questo era l'orizzonte. Ulisse trasforma questi istinti primordiali in altrettanti miti. La violenza cieca diventa il ciclope, la lussuria diventa la maga Circe, l'ebbrezza diventano i lotofagi, guidato dall'indovino Tiresia scende negli Inferi e racconta anche la morte. Dopo di lui il mondo è raccontabile.» «Intervista di Maurizio Zuccari con Sebastiano Vassalli: Lo scrittore e la storia», dicembre 2003, tratta dal sito http://www.ragionamentidistoria.it

${ }^{22}$ Sebastiano Vassalli, Cuore di pietra, op. cit., p. 286.

${ }^{23}$ Ibidem, p. 32.
} 
volta per sempre dal carattere dei singoli personaggi e dalla professione esercitata il più delle volte ereditata - poiché

il passato non riesce mai a passare del tutto e il presente non è mai davvero presente ${ }^{24}$.

Nonostante questo, però, il tempo ad un certo punto si mette inaspettatamente in movimento durante la Prima Guerra mondiale, quando i personaggi si ritrovano in situazioni drammatiche e i destini degli uomini o si concludono ex abrupto, oppure attraversano cambiamenti inaspettati. Dopo l'intermezzo fascista poi arriva una nuova epoca: muore l'ultimo socialista e in una regione con cinquanta chiese tutto ad un tratto manca il parroco. Proprio in questo romanzo Vassalli si concede nel modo più evidente i vantaggi del narratore omerico onnisciente, del poeta epico che registra non solo ciò che è palese, ma anche ciò che è rimasto nascosto e che con l'aiuto di Calliope, la quale conosce le storie e le guerre di tutti gli uomini, riesce a udire anche il lamento delle ninfe che arriva dalle cime dei monti.

Con il romanzo Archeologia del presente (2001) Vassalli è tornato ancora una volta al passato recente, al suo personale ieri. Il romanzo si lega così idealmente, come una sorta di capitolo sciolto, sia al più vecchio $L$ 'oro del mondo, sia agli ultimi capitoli dei romanzi Cuore di pietra e Le due chiese. Vassalli ha scritto in questo lavoro il suo libro più personale: la generazione che vi ha rappresentato è la sua propria generazione, la generazione dei sessantottini. Il titolo del libro è preciso: ciò di cui vi si parla nel 2001 è ancora il «presente», ma un presente il cui slancio si è completamente esaurito e che è necessario estrarre con fatica dai ricordi, per quanto recenti essi siano. Nello sforzo di illustrare il proposito che perseguiva con questo libro, in un'intervista Vassalli ha ricordato Bouvard e Pecuchet di Flaubert, due archeologi che scavavano ostinatamente nel mito del loro presente, quel mito che portava il nome di «progresso».

Il narratore - un architetto non troppo facoltoso, abituato ad adeguarsi sempre alle circostanze - rievoca la storia dei suoi due amici Leo e Michela, che si sono fatti carico di un compito che hanno inteso come il mandato dell'epoca: cambiare il mondo. Entrambi contano su una buona sicurezza economica e questo, in un momento di particolare amarezza, induce il narratore a un commento caustico:

I veri poveri pensano a se stessi. Sono gli altri, i benestanti, che vorrebbero fargli fare la rivoluzione ${ }^{25}$.

Leo e Michela dedicano il loro impegno militante a ogni cosa che i tempi richiedano loro: lottano per i diritti delle donne, sulla scorta della lezione di Foucault ospitano nella loro casa un pazzo per rompere le barriere tra i sani e i malati di mente, nella scuola in cui insegnano introducono l'antididattica sull'esempio di don Milani (come conseguenza la maggior parte dei studenti di Leo viene bocciata alla maturità), protestano contro i cacciatori, sono vegetariani, fanno un viaggio in Cina e si entusiasmano per le conquiste culturali del maoismo, partecipano a campeggi pacifisti, si esaltano per l'ecologia e aprono la loro casa a immigrati clandestini.

${ }^{24}$ Sebastiano Vassalli, Le due chiese, Torino, Einaudi 2010, p. 40.

${ }^{25}$ Sebastiano Vassalli, Archeologia del presente, Torino, Einaudi 2001, p. 20. 
Adottano due bambini e uno di loro, con gravi problemi di disadattamento sociale, un giorno li uccide.

La vita di Leo e Michela, piena di entusiasmi mai placati e di altrettante inevitabili frustrazioni, si conclude quindi con una morte assurda. Il disincantato narratore fin dall'inizio della narrazione constata:

Tutto, in superficie, ribolliva e si agitava in modo convulso; ma dietro al clamore delle dichiarazioni e dei proclami, dietro al frastuono degli scontri di piazza e degli attentati, dietro al sangue delle vittime e all'accavallarsi delle notizie il vecchio mondo celebrava i suoi riti di sempre, e non c'era assolutamente niente che potesse turbarlo, o intralciarlo... ${ }^{26}$

Nonostante tutta l'ironia, però, il romanzo termina con una sincera nenia per questa generazione perduta:

Adesso che lui e Michela non ci sono più, chi ci salverà? Chi affronterà le ingiustizie, ad una ad una, come don Chisciotte affrontava i mulini a vento? Chi eliminerà le scorie radioattive e le malattie iatrogene? Chi pulirà l'atmosfera, e ci restituirà le stagioni? Chi provvederà ai cani e ai gatti abbandonati? Chi difenderà gli zingari e gli immigrati clandestini? ${ }^{27}$

È la stessa nenia per i folli redentori che era risuonata già alla fine del romanzo Marco e Mattio.

Per tre volte Vassalli è tornato ad un passato molto remoto. La prima volta nel romanzo La notte del lupo (1998), nel quale aveva rielaborato la storia di YoshuaGesù e di Giuda. Un anno dopo aveva pubblicato un interessante racconto sul passato etrusco della Roma augustea, intitolato Un infinito numero (1999). Nel 2014 infine ha pubblicato il romanzo Terre selvagge, nelle quali ha rievocato la disfatta dei Cimbri nell'anno 101 a.C. ai Campi Raudii, nella regione dell'attuale Piemonte - lembo particolare della «grande e misteriosa Europa».

Soffermiamoci, per chiudere, sul romanzo Un infinito numero, un saggio straordinariamente convincente dell'arte narrativa di Vassalli. Il romanzo è composto in modo eccellente (l'ingegnoso arabesco della narrazione si chiude elegantemente in un cerchio) ed è ancora una volta popolato da decine di figure dai caratteri definiti con precisione, sebbene spesso siano solo tratteggiati con la tecnica allusiva dello schizzo ad acquerello.

Anche nel caso di questo romanzo è molto difficile (e di certo anche superfluo) tracciare una linea di demarcazione tra la libera fabulazione e la documentazione di fatti verificabili. È chiaro che Vassalli si orienta con grande sicurezza nel materiale storico che ha utilizzato, ma questo materiale già di per sé non è troppo attendibile. Alla sua base ci sono antiche relazioni che narrano fatti trasformati in leggenda dal solo punto di vista dei Romani (l'unica opera romana sul passato etrusco, i venti libri scritti in greco dal titolo Tyrrhenika, del dotto imperatore Claudio, sono andati irrimediabilmente perduti ed è diventato determinante l'ingenuo racconto di Livio). Vassalli ha quindi avuto mano libera per costruire il suo quadro con la scelta - o la contaminazione - di una tradizione spesso contraddittoria. I suoi Etruschi (che nella

\footnotetext{
${ }^{26}$ Ibidem, p. 29.
}

${ }^{27}$ Ibidem, p. 171. 
loro lingua si chiamavano Rasna o Rasenna) sono discendenti dei conquistatori Lidi guidati dal mitico Enea, un corpulento e puzzolente tagliagole (era stato Erodoto ad attribuire l'origine Lidia agli Etruschi), i quali si mescolarono con gli antichi abitanti delle coste italiane (gli Etruschi erano considerati invece popolazione autoctona da Dionigi di Alicarnasso). Vassalli per contro è d'accordo in tutto e per tutto con le opinioni dei moderni ricercatori, che descrivono gli Etruschi come eccellenti navigatori, costruttori e artisti inimitabili, come un popolo che viveva del mito e di rappresentazioni religiose collettive, un popolo a cui era del tutto estraneo l'individualismo, il pragmatismo e la tendenza all'epicureismo propri dei romani.

Vassalli è altrettanto ricettivo nei confronti della realtà del mondo romano: attribuisce all'imperatore romano Ottaviano Augusto e a Mecenate fisionomie plastiche - e senza dubbio «possibili» - (sebbene si possa ritenere che alcuni tratti di Mecenate li abbia presi in prestito dal Trimalcione di Petronio). Descrive il personaggio centrale della storia, lo schivo e introverso Virgilio, nella tradizione che ne vedeva l'autoritratto nel modesto contadino delle sue Georgiche (in Italia questa immagine del poeta che aspira a una vita ritirata era stata fissata da Leopardi nei Detti memorabili di Filippo Ottonieri, una delle sue Operette morali). E come già nei suoi precedenti romanzi, inserisce anche queste sue creazioni nella trama della storia intessuta dalla «grande politica»: dalla guerra civile e dalla disgregazione economica e amministrativa del paese o dall'opera di riforma di Ottaviano Augusto, che col pugno di ferro instaurò un'amministrazione controllata militarmente e trasformò la Roma di mattoni nella Roma di pietra.

Il romanzo di Vassalli, insomma, non ha niente di meno «verosimile» rispetto ad altri romanzi storici che attingono da questo periodo. È però evidente che a Vassalli non interessava essere lodato per questo. Ancora una volta, voleva soprattutto narrare una storia, la sua storia (questo è sottolineato esplicitamente nel passaggio introduttivo e in quello conclusivo, dove il narratore si intrattiene col suo personaggio per scambiarsi alla fine di posto con lui): la storia sui sogni di un'epoca.

Vassalli è soprattutto affascinato dal fatto che gli Etruschi, un popolo straordinariamente evoluto culturalmente e pratico della scrittura, non abbiano lasciato nessuna letteratura. Nel suo romanzo propone una spiegazione di questo mistero: gli Etruschi vivevano nel tempo chiuso del mito, in un tempo senza futuro, un tempo in cui ogni cosa era compiuta e costantemente presente: nel quale

ogni avvenimento, da quelli apparentemente insignificanti come la caduta di una foglia o la morte di un passero, a quelli che cambiano il paesaggio di un'intera regione o il destino di un popolo, si colloca in una catena infinita di eventi, e deve essere messo in rapporto con tutto quello che lo precede e lo segue ${ }^{28}$.

Vassalli fa intraprendere ai suoi tre pellegrini, Virgilio, Mecenate e Timodemo, un viaggio iniziatico nel sotterraneo del tempio di Mantus, nel quale questi eletti vivono centinaia di vite che nell'insieme sono la vita unica del collettivo etrusco,

${ }^{28}$ Sebastiano Vassalli, Un infinito numero, Torino, Einaudi 1999, p. 113. -43 - 
una polifonia di gioie, di sofferenze e di speranze che rivivono in continuazione. Gli Etruschi, per i quali il vissuto del proprio essere collettivo era accessibile in ogni istante nell'eterno presente del mito, secondo l'interpretazione di Vassalli non sentivano il bisogno di fissare questo vissuto con le parole; sapevano che con la scrittura la vita esce dal tempo, che la scrittura falsifica le storie e poi le mortifica; che la scrittura è la morte. Secondo Vassalli essi conoscevano la poesia ed erano capaci di goderne: il loro cantore Aveles conosceva l'Eneide già prima di Virgilio; era però uno dei rapsodi orali che creavano la poesia con la loro voce iniziando ogni volta da zero, qui e ora, in quel presente e da quel presente in cui viveva il mito.

La cultura degli Etruschi si estingue nel momento in cui lo straniero Virgilio traduce questo canto nella propria lingua e lo scrive su un rotolo di carta. Trasferisce così il mito vivo dentro le strutture pragmatiche di una civiltà del tutto differente, una civiltà in cui la letteratura ha una funzione completamente diversa. La civiltà romana - indica Vassalli -, quella civiltà di cui siamo eredi, ha annullato definitivamente l'omologia della vita e della parola mitica. La letteratura non ha perduto il suo credito, ma i motivi della sua importanza si sono trasformati.

Il grammatico Timodemo incontra la letteratura nella biblioteca di Virgilio, e ne è stupefatto: per quattro anni rimane immerso nella lettura e si abitua incantato «a guardare il mondo con cento occhi, anziché con i miei due soli, e a sentire nella mia testa cento pensieri diversi, anziché il mio solo pensiero»; la letteratura gli insegna a diventare «consapevole di se stesso e degli altri”" ${ }^{29}$. La letteratura è così - nella tradizione occidentale - lo strumento per una migliore conoscenza del mondo; tuttavia una conoscenza mai compiuta, che di fatto rimane solo docta ignorantia, e non rende mai possibile quel colpo d'occhio che d'un tratto fa comprendere l'insieme, quel sapere completo che era mediato dal mito.

In questa nuova prospettiva la letteratura è nello stesso tempo qualcosa di estremamente artificioso, qualcosa che ha le proprie regole e che si può portare alla perfezione solo a costo di uno sforzo immenso. E perciò è anche necessario porsi la domanda di cosa può parlare la poesia, cosa è degno di una tale fatica. Nessuno lo sa meglio di Virgilio, che cesella la sua epopea per interi lunghi anni. Egli spiega anche a Timodemo qual è lo scopo della letteratura nella nuova epoca:

La poesia deve mostrarci la parte migliore dei nostri sentimenti, cosi come la pittura e la scultura ci mostrano l'armonia dei nostri corpi: e, se anche qualche volta ne rappresentano le deformità, lo fanno soltanto per dare ancora più risalto alla bellezza... per guardarci come siamo fatti davvero, bastano gli specchi! ${ }^{30}$

Una letteratura come quella creata da Virgilio non rivendica nessuna veridicità, che era un attributo naturale del mito. Il suo scopo non è la verità, ma la bellezza. Il consumatore ideale di questa letteratura è quindi un edonista del tipo di Mecenate. Creare la bellezza è certo difficile, però è più facile che dire la verità; è una questione di talento e di tecnica, e quindi è un compito alla portata degli uomini. E così sulla

${ }^{29}$ Ibidem, p. 31.

${ }^{30}$ Ibidem, p. 202. 
riva del Tevere - col fattivo sostegno di Mecenate - ad un tratto compaiono sciami di poeti, più di quanti se ne siano mai visti prima.

Meglio di tutti però ha compreso il valore della letteratura Ottaviano. È proprio lui a chiedere che la poesia, definitivamente sottratta al mito, tenti di ricrearlo e vi includa Roma, «le sue origini, le origini del suo principe ${ }^{31}$. Ha capito meglio di chiunque altro che a questi poeti non stava più a cuore la verità e che in nome della bellezza avevano imparato a modificare la realtà. Il vero nome del «mito» che egli ha in mente è proprio la «storia», quella Storia con la lettera maiuscola che dettano i vincitori (e che - aggiungiamo - lo stesso Vassalli aveva rifiutato di servire già nei suoi primi romanzi). Perché in questa nuova funzione la parola scritta non porta più la morte, ma la vita. È servitrice della dea Fama, la massima divinità del mondo moderno, l'essere invincibile e instancabile «che tiene uomini sotto il suo potere, propagando con voce di tuono ogni genere di notizie», ${ }^{32}$ le più evidenti verità come le più spudorate menzogne.

Tutta l'opera di Vassalli si sviluppa attorno a due temi centrali: il primo è la storia, il secondo la letteratura.

Per quanto Vassalli - scettico, agnostico, nemico di tutti i progetti utopistici possa essere allergico a qualunque idealismo naif, la sua fede nel senso del «raccontare» resta incrollabile. Ricorda a più riprese che raccontare è la nostra unica memoria: l'unica difesa contro la caduta nel nulla e nel silenzio. Verbis expressis ne ha parlato ad esempio in una intervista con Giovanni Tesio:

Il mestiere dello scrittore consiste nel raccontare storie. Così era ai tempi di Omero e così è ancora oggi. È un mestiere antico come il mondo, che risponde a una necessità degli esseri umani, ad un loro bisogno fondamentale: quello di raccontarsi. Finché ci saranno nel mondo due persone, ci sarà chi racconta una storia e ci sarà chi ascolta una storia. [...] Senza la memoria del passato che è all'origine di ogni racconto, il nostro percorso di civiltà sarebbe ancora fermo da qualche parte nella notte dei tempi ${ }^{33}$.

Proprio per questo il suo volgersi al passato è di una straordinaria generosità. Le vicende che egli racconta si svolgono su una scala temporale che va dall'antichità ai giorni nostri e il loro scenario ha innumerevoli varianti. Nella stessa intervista ha detto con motivato orgoglio:

Forse nessuno se ne è accorto, ma io ho finito per raccontare l'Italia fuori dai particolarismi e dai localismi. L'ho raccontata a nord, a sud, a est e a ovest ${ }^{34}$.

${ }^{31}$ Ibidem, p. 52.

32 Ibidem, p. 208.

33 Giovanni Tesio - Sebastiano Vassalli, Un nulla pieno di storie. Ricordi e considerazioni di un viaggiatore nel tempo, Novara, Interlinea 2010, p. 77.

${ }^{34}$ Ibidem, p. 90. 
Già di fronte alla distruzione dei topoi tradizionali e dei principi mimetici operata dalla neoavanguardia il discorso sul passato gli era sembrato qualcosa che potesse restituire un senso all'impegno letterario. La neoavanguardia forse era stata espressione di quella moderna confusione nella quale una voce grida più forte di un'altra e la copre, però lo sguardo rivolto all'indietro - una profonda anamnesi consentiva di raggiungere immagini che non solo orientano una luce inaspettata anche su eventi odierni, ma permettono soprattutto di riallacciare un rapporto profondo con i fatti della vita in genere:

Forse ci sono dei periodi, nella nostra storia, in cui per guardare avanti bisogna voltarsi. Come ai tempi di Omero e come oggi. Achille, Ettore e Ulisse avevano qualcosa da dire a chi viveva mille anni dopo di loro e hanno qualcosa da dire ancora a noi, dopo che sono passati altri tremila anni. Qualcosa che né la televisione né il web né i vicini di casa saprebbero dirci ${ }^{35}$.

La storia in quanto cronologia di eventi collettivi ovviamente per Vassalli come abbiamo potuto constatare più volte - è una falsa costruzione. Come ha sempre ricordato, la storia in realtà non è nient'altro che una muta corrente che trasporta le vicende umane ${ }^{36}$. La storia nella dimensione enciclopedica secondo lui in realtà non è mai esistita, «non è mai esistita la storia di una vicenda comune di tutti gli esseri umani che progrediscono nel tempo» ${ }^{37}$.

Anche per questo il compito del narratore non è offrire una riproduzione per quanto possibile verosimile dei fatti (e a questo riguardo in linea di principio non è d'accordo neanche col suo modello Alessandro Manzoni, il quale preferiva il «vero storico» al «vero d'invenzione»). E in genere non deve cercare di estrapolare massime filosofiche, politiche o morali dal passato.

Il narratore di Vassalli non scrive romanzi à thèse - come fa ad esempio Umberto Eco - al contrario, disegna immagini nelle quali non è nascosta nessuna verità atemporale e nessuna sentenza esortativa. La ricostruzione storica dei fatti per quanto garantita da un'accurata documentazione - resta per lui semplice materiale narrativo. Questo narratore non si accosta alla massa dei ricordi storici come interprete, quanto piuttosto come controrelatore. In un'intervista con Nicola Fano una volta Vassalli lo ha formulato con nettezza:

Un romanzo non descrive la realtà. Semmai la sfida. No, non per crearne necessariamente un'altra, ma per sfidarla in senso stretto, per vedere come reagisce alla provocazione ${ }^{38}$.

\footnotetext{
${ }^{35}$ Sebastiano Vassalli, Terre selvagge, Milano, Rizzoli, 2014, p. 295-296.

${ }^{36}$ Cfr. Giovanni Tesio, „Sebastiano Vassalli, un narratore di storie tra Omero e il signor B. », in Giovanni Tesio - Sebastiano Vassalli, Un nulla pieno di storie. Ricordi e considerazioni di un viaggiatore nel tempo, ed. cit., p. 125: "Se la storia non vince il tempo, ne registra però le azioni e ne verbalizza i movimenti di scena. Stabilito che il tempo non è che l'illusione suprema di un'impossibile eternità, la storia non ha altro compito che quello di annotare le chimere, di chiosare l'assurdo, di rammentare le apparenze sfilacciate di una condizione che non conosce riscatto...»

37 «Intervista di Maurizio Zuccari con Sebastiano Vassalli: Lo scrittore e la storia», dicembre 2003 , trattta dal sito http://www.ragionamentidistoria.it

${ }^{38}$ L'Unità, 25. 1. 1990.
} 
La grande storia per lui è rilevante solo come serbatoio di miti - un tempo autentici e in seguito falsi. Nel flusso del tempo solamente i miti hanno una forza davvero formativa. Agli albori delle nostre civiltà sono perfino riusciti a creare un'entità unica dai collettivi nazionali: un ruolo simile ad esempio è stato svolto dal mito nella vita degli Etruschi. I miti moderni non hanno più una forza così agglomerante, ma tuttavia alcuni di essi hanno svolto a lungo e con successo la loro funzione originale. A Vassalli interessano soprattutto due di questi miti: il cristianesimo cattolico e il socialismo. Il mito cristiano ha vissuto sempre una vita piena nelle personalità dei pazzi e dei santi, come Mattio o suor Partenope, però è anche potuto degenerare nelle pratiche inquisitorie, le pratiche che hanno annientato Antonia Spagnoletti. Vassalli attribuisce lo stesso carattere doppio anche ai miti della sinistra (ai quali da giovane aveva aderito anche lui). La loro ingenuità e il loro essere controproducenti sono crudelmente caricaturizzati in Archeologia del presente, mentre Le due chiese comincia e finisce con il ricordo dell'Internazionale, espressione emblematica del sogno socialista di fraternità e di uguaglianza. I miti possono essere anche fabbricati per scopi funzionali: in questo modo Vassalli percepisce già il mito nazionale posto da Virgilio nella sua Eneide. Anche gli pseudo-miti naturalmente sono capaci di dare un orientamento alle nostre vite, per quanto funesto: vedi il comunismo caricaturizzato in Mareblù, il fascismo rappresentato ne L'oro del mondo o il mito mafioso della «famiglia» raffigurato ne Il cigno.

Vassalli riconosce che la letteratura ha avuto fin troppo spesso parte attiva nel consolidamento di questi pseudo-miti, ma è la letteratura che egli rifiuta. Come già detto, egli ha una ricetta semplice per non seguire queste tracce: non descrive «la Storia», ma racconta «le storie». Il suo universo picaresco è normalmente popolato da numerosi attori, le cui storie si somigliano tra loro - «le storie umane, pur cambiando dei particolari, continuano a ripetersi $\rangle^{39}$ - ma nello stesso tempo sono sempre nuove e diverse. La vera, concreta storia per Vassalli allora non è altro che l'amalgama di queste storie, la loro somma e la loro ipostasi. Molto spesso sono storie di ignoti, di quelli che gli storici di professione - i portavoce dei vincitori non prendono in considerazione e che scorrono via assieme al tempo per perdersi nell'oblio. Soltanto attraverso le singole vicende umane si può comprendere davvero il passato, solo tramite esse si possono capire il suo potenziale universale e le sue strutture profonde. «Io sono un animale di profondità, » constatava Vassalli.

Mi interessano i movimenti profondi: per esempio quello che ho cercato di raccontare nel romanzo Un infinito numero. O nel romanzo La Chimera... Credo che la letteratura, oltre che a raccontare il mondo, deve servire a capirlo ${ }^{40}$.

${ }^{39}$ Terre selvagge, op. cit., p. 29.

${ }^{40}$ Giovanni Tesio - Sebastiano Vassalli, Un nulla pieno di storie. Ricordi e considerazioni di un viaggiatore nel tempo, op. cit., p. 57. 
Il suo rifiuto delle «grandi vicende» - il suo «nichilismo», come lo chiama Hanna Serkowská - ha anche il suo lato positivo ${ }^{41}$. Proprio nel portare alla luce il substrato più profondo della storia Vassalli vede un compito della letteratura che abbia un senso e le consenta di perpetuarsi. Così lo ha formulato ad esempio nell'introduzione al romanzo Io Partenope, apostrofando la sua eroina:

È lì che, finalmente, posso avvicinarla, e parlarle, in un tempo che non è più il suo tempo e non è ancora il mio: è il tempo della letteratura, dove tutto, o quasi tutto, è possibile. Dove ci si può incontrare scavalcando i secoli, che in natura non esistono, nell'eterno presente delle vicende umane $^{42}$.

Alla postmoderna sfiducia nei confronti della storia, Vassalli ha contrapposto la fiducia nell' «eterno presente» delle vicende umane.

\section{BIBLIOGRAFIA}

\section{Opere citate di Sebastiano Vassalli}

3012. L'anno del profeta (1995), Torino, Einaudi.

Abitare il vento (1980), Torino, Einaudi.

L'alcova elettrica (1986), Torino, Einaudi.

Archeologia del presente (2001), Torino, Einaudi.

Arkadia: carriere, caratteri, confraternite degli impoeti d'Italia (1983), Bergamo, El Bagatt.

L'arrivo della lozione (1976), Torino, Einaudi.

La chimera (1990), Torino, Einaudi.

Il cigno (1993), Torino, Einaudi.

Il confine. I cento anni del Sudtirolo in Italia (2015), Milano, Rizzoli.

Cuore di pietra (1996), Torino, Einaudi.

Disfaso (1968), Roma, Trevi.

Le due chiese (2010), Torino, Einaudi.

Un infinito numero: Virgilio e Mecenate nel paese dei Rasna (1999), Torino, Einaudi.

Io, Partenope (2015), Milano, Rizzoli.

Gli italiani sono gli altri: viaggio (in undici tappe) all'interno del carattere nazionale italiano (1998), Milano, Baldini \& Castoldi.

L'italiano (2007), Torino, Einaudi.

\footnotetext{
${ }^{41}$ Hanna Serkowska, «Sebastiano Vassalli: da abitante del vento a seguace del nulla», Cahiers d'études italiennes (Grenoble), 2009, n. 9, p. 82: «Il nichilismo di marca vassalliana non è di carattere nirvanico, autosufficiente, contemplativo, bensì rimarca l'atteggiamento disincantato e disilluso di chi prova tuttavia un autentico interesse, preoccupazione e impegno per le cose di questo mondo e di questa società.»

${ }^{42}$ Sebastiano Vassalli, Io Partenope, Milano, Rizzoli 2015, p. 12.
} 
Il millennio che muore (1972), Torino, Einaudi.

Marco e Mattio (1992), Torino, Einaudi.

Mareblù (1982), Milano, Mondadori.

Narcisso (1968), Torino, Einaudi.

La notte della cometa. Il romanzo di Dino Campana (1984), Torino, Einaudi.

La notte del lupo (1998), Milano, Baldini \& Castoldi.

L'oro del mondo (1987), Torino, Einaudi.

Sangue e suolo. Viaggio fra gli italiani trasparenti (1985), Torino, Einaudi.

Stella avvelenata (2003), Torino, Einaudi.

Tempo di massacro (1970), Torino, Einaudi.

Terre selvagge (2014), Milano, Rizzoli.

\section{Saggi}

CiCALA Roberto (2012), La sperimentazione del giovane Vassalli, in: CiCALA Roberto, Inchiostri indelebili. Itinerari di carta fra bibliografie, archivi e editoria, Milano, Educatt, p. 215-248.

CiCAla Roberto, TESIO Giovanni (a cura di) (2003), La Chimera. Storia e fortuna del romanzo di Sebastiano Vassalli, Novara, Centro Novarese di Studi Letterari - Interlinea.

LA MENDOLA Velania (2011), «L'onorevole» e «il giullare di provincia». Incontro e scontro letterario tra Sciascia e Vassalli, Microprovincia 49, (La parola e le storie di Sebastiano Vassalli. Omaggio per i settant'anni dello scrittore), p. 191205.

Mondo Lorenzo (2015), Questi piemontesi. Profili di scrittori italiani tra Otto e Novecento, Firenze, Leo S. Olschki.

NESI Cristina (2005), Sebastiano Vassalli, Fiesole, Cadmo.

SERKOWSKA Hanna (2009), Sebastiano Vassalli: da abitante del vento a seguace del nulla, Cahiers d'études italiennes, 9, p. 81-90.

Tesio Giovanni - VASSALli Sebastiano (2010), Un nulla pieno di storie, Novara, Interlinea.

VASSALli Sebastiano, Lollini Attilio (1991), Belle lettere, Torino, Einaudi.

\section{Sitografia}

http://www.ragionamentidistoria.it 
- 50 - 